\title{
Thyrotoxic Crisis Associated with Gestational Trophoblastic Disease
}

\author{
Carlos Eduardo David de Almeida, TEA ${ }^{1}$, Erick Freitas Curi, TSA ${ }^{2}$, Carlos Roberto David de Almeida, TEA ${ }^{3}$, \\ Denise Fernandes Vieira ${ }^{4}$
}

Summary: Almeida CED, Curi EF, Almeida CRD, Vieira DF - Thyrotoxic Crisis Associated with Gestational Trophoblastic Disease.

Background and objectives: Human chorionic gonadotropin (HCG) and thyrotrophic hormone (TSH) have analogies in their structures, as well as in their receptors. The high levels of HCG seen in gestational trophoblastic diseases may induce secondary hyperthyroidism. The objective of this report was to present a case in which the administration of iodinated contrast triggered a thyrotoxic crisis.

Case report: Patient with complete hydatidiform mole who was admitted to the operating room with severe vaginal bleeding after a tomographic exam with iodinated contrast. During anesthetic induction, the patient presented symptoms compatible with thyrotoxic crisis.

Conclusions: The incidence of severe presentations associated with gestational trophoblastic disease tends to decrease with early diagnosis. Still, the anesthesiologist should be aware of the possibility of those patients developing thyrotoxic crisis.

Keywords: Hydatidiform mole; Intraoperative Complications; Hyperthyroidism; lodopyridones.

\section{INTRODUCTION}

Gestational trophoblastic disease (GTD) is characterized by abnormal proliferation of the trophoblastic epithelium (cytotrophoblast, syncytiotrophoblast, and intermediate trophoblast). It has the potential for local invasion or metastasis.

The World Health Organization classifies GTD according to the anatomoclinical characteristics: malignant neoplasia (gestational choriocarcinoma, placental site trophoblastic tumor, and epithelial trophoblastic tumor), malformation of chorionic vilosities that predispose the development of malignant neoplasia (hydatidiform mole and its subtypes), and benign entities (exaggerated placental site reaction and placental site nodule).

Received from Universidade Federal do Espírito Santo (UFES); Hospital Universitário Cassiano Antônio Moraes (HUCAM), Brazil.

\footnotetext{
1. TEA MEC/SBA from Universidade de São Paulo (USP); Assisting Physician of the Serviço de Anestesiologia of HUCAM/UFES

2. President of the Sociedade de Anestesiologia do Espírito Santo (SAES); Co-responsible for the CET Integrado HUCAM/HAFPES; Assisting Physician of the Serviço de Anestesiologia of HUCAM/UFES

3. TEA MEC/SBA from UFES; Assisting Physician of the Serviço de Anestesiologia of HUCAM/UFES

4. R3; Anesthesiology Resident at the CET/SBA Integrado HUCAM/HAFPES

Submitted on November 4, 2010.

Approved on January 31, 2011.

Correspondence to:

Dr. Carlos Eduardo David de Almeida

Av. Cesar Helal, 1.181, apt 1.903

Praia do Suá

29052-230 - Vitória, ES, Brazil

E-mail: cedalmeida@terra.com.br
}

Normal or pathological trophoblast produces human chorionic gonadotropin ( $\mathrm{HCG}$ ). Studies indicate the analogy between HCG and thyrotrophic hormone (TSH), as well as among their receptors ${ }^{1}$. The high levels of HCG seen in GTD may induce secondary hyperthyroidism.

Administration of iodine-containing medications may trigger a thyrotoxic crisis, since the trophoblastic production of $\mathrm{HCG}$ is not inhibited by the production of thyroid hormones.

It is presented here the case report of a thyrotoxic crisis in a patient with complete molar pregnancy associated with the use of iodinated contrast for a tomographic exam.

\section{CASE REPORT}

This is a 13 year-old caucasian patient, $45 \mathrm{~kg}$, admitted to the gynecology and obstetrics service with complaints of amenorrhea, abdominal pain, and vaginal bleeding. She was tachypneic, tachycardic (112 bpm), hypertensive (165 x $92 \mathrm{mmHg}$ ), with a mucous membranes pale, and dehydrated. Laboratorial exams upon admission were: $9.9 \mathrm{mg} . \mathrm{dL}^{-1}$ of hemoglobin, hematocrit $31.3 \%$, leukocytes 11,800 with $6 \%$ granulocytes, 254,000 platelets with coagulogram within normal limits, and TSH 0.009 (reference 0.35 to 5.5). The levels of HCG were higher than 400,000 UI.L-1. Ultrasound showed uterine volume of $1,780 \mathrm{~cm}^{3}$ with multiple cystic vesicles compatible with hydatidiform mole.

She was admitted in the operating room on the same day of hospitalization to undergo an uterine curettage due to severe vaginal bleeding after a chest, abdominal, and pelvic $\mathrm{CT}$ with iodinated contrast.

General balanced anesthesia was performed with intravenous induction with fentanyl $\left(10 \mu \mathrm{g} \cdot \mathrm{kg}^{-1}\right)$, propofol $\left(2 \mathrm{mg} \cdot \mathrm{kg}^{-1}\right)$, 
and atracurium $\left(0.5 \mathrm{mg} \cdot \mathrm{kg}^{-1}\right)$. After orotracheal intubation the patient developed sinus tachycardia (170 bpm), hypertension $(160 \times 120 \mathrm{mmHg})$, hypercarbia $(52 \mathrm{mmHg})$, and acute pulmonary edema. She was treated with esmolol and intravenous sodium nitroprusside $75 \mu \mathrm{g} \cdot \mathrm{kg}^{-1} \cdot \mathrm{min}^{-1}$ and $2.0 \mu \mathrm{g} \cdot \mathrm{kg}^{-1} \cdot \mathrm{min}^{-1}$ respectively with satisfactory response. She was transferred to the intensive care unit hemodynamically stable, being discharged to the regular ward after two days.

\section{DISCUSSION}

It has been estimated that there is one case of hydatidiform mole (HM) for every 1,000-2,000 pregnancies in the Western World, and among them trophoblastic disease is the most common ${ }^{2}$. Brazilian statistics are based on data from teaching hospitals and it can translate into a falsely elevated incidence ${ }^{3}$.

Risk factors include maternal age, history of hydatidiform mole, viral infections, nutritional status, number of pregnancies, and oral contraception. The extremes of age also show an increased risk, and women over 40 years of age have a 10-times higher risk than women between 22 and 40 years of age.

Hydatidiform mole can be divided in partial and complete. In complete HM the embryo, umbilical cord, and membranes do not develop. Hydropic dilation in all vilosities, as well as formation of a central pool full of liquid are observed. Red blood cells or fetal blood vessels are not observed in the vilosities; however, it is possible that $\mathrm{RH}$ negative gravidas will develop sensitization. Complete HM has exclusively paternal chromosomes (androgenesis), and most are 46,XX. The empty egg is fertilized by a $23, X Y$ spermatozoid, and duplication of the paternal genome occurs. Approximately $5 \%$ to $10 \%$ are $46, X Y$ due to the fertilization of an egg without genetic load by two spermatozoids ( $\mathrm{X}$ and $\mathrm{Y}$ ). Complete HM evolves to malignant types in $20 \%$ of cases.

In partial HM one can see microscopically an embryo with malformations. In $90 \%$ of cases it originates in a normal egg fertilized by two spermatozoids resulting in a triploid cell $(69, X X X$ or $69, X X Y)$. Only $5 \%$ of cases evolve to malignant forms ${ }^{4}$.

Vaginal bleeding is the most common clinical sign (84\%) associated or not with menstrual delay. Vomiting is present in $28 \%$ of cases and can be refractory to treatment. Hyperemesis gravidarum is seen more commonly in cases of hydatidiform mole with high HCG levels ${ }^{5}$.

In advanced cases, exaggerated uterine growth is a common complaint. The presence of tecaluteinic cysts due to ovarian hyperstimulation secondary to high HCG levels is common. Pregnancy-associated hypertension is present in approximately $30 \%$ of cases of complete HM.

The increase in uterine volume associated with high HCG, and consequently progesterone, levels may favor aspiration of gastric contents during anesthesia under sedation or inadequate airways control. In the absence of contraindications, spinal anesthesia is preferred for uterine emptying. Severe bleeding and increased risk of uterine perforation are other complications of the surgical procedure.

In approximately $2 \%$ of cases trophoblastic embolization is observed, which causes tachypnea, thoracic pain, and tachycardia. Thus, in the presence of indicative symptoms radiological tests should be performed to prove the presence of HM.

High HCG levels stimulate the thyroid gland with suppression of pituitary TSH release. Serum concentrations above $200,000 \mathrm{mlU}^{\mathrm{mL}} \mathrm{m}^{-1}$ have been demonstrated to suppress TSH (lower or equal to $0.2 \mathrm{mlU}^{\mathrm{mL}-1}$ ) in $67 \%$ of cases, and levels above $400,000 \mathrm{mlU}^{\mathrm{mL}} \mathrm{m}^{-1}$ promote suppression in $100 \%$ of cases ${ }^{6}$. Trophoblastic HCG production is not inhibited (negative feedback) by the increased levels of thyroid hormones.

Clinical hyperthyroidism can be observed in less than $10 \%$ of cases. Symptoms include: tachycardia, hypertension, tremors, tachypnea, weight loss, heat intolerance, muscle weakness, diarrhea, nervousness, and hyper-reactive reflexes.

The use of iodinated substances can trigger a thyrotoxic crisis (Jod-Basedow phenomenon) ${ }^{7}$. Besides drugs, the use of disinfectants, antiseptics, and iodinated contrast agents may trigger thyrotoxic crisis. Amiodarone is the drug more often correlated with thyroid stimulation. A $100 \mathrm{mg}$ pill contains 250 times the recommended daily dose of iodine ${ }^{8}$.

Under physiological conditions, increased serum levels of iodine causes and increase in transport and in iodine pool resulting in inhibition of thyroid hormones synthesis and selfregulatory inhibition of iodine transportation (Wolff-Chaikoff phenomenon) ${ }^{9}$. Thus, the excess of iodine and absence of a regulatory system can lead to persistent increase in the production of thyroid hormone and thyrotoxicosis.

In the case reported here, the use of iodinated contrast to perform the scans before surgical intervention could have contributed to the development of thyrotoxic crisis. The use of prophylactic anti-thyroid drugs in exams with iodinated contrast is controversial ${ }^{10}$.

The reduction of thyroid hormones is the initial step for treatment of thyrotoxicosis. Thionamides (propylthiouracil, metimazole) are the antithyroid medications used more often. They inhibit thyroid peroxidase therefore inhibiting the incorporation of iodine into thyroglobulin. Propylthiouracil (PTU) has the additional mechanism of inhibiting the peripheral conversion of T4 to T3.

The choice of antithyroid drug depends on several factors. Metimazole has dose-dependent side effects. Cases of hepatotoxicity are less severe, and it is administered in a single daily dose. Propylthiouracil may be the drug of choice in pregnancy and lactation due to reduced placental crossing and lower levels in maternal milk when compared to PTU. In more severe cases, such as thyrotoxic storm, PTU should be the drug of choice because, in addition to the inhibitory effects on the release of thyroid hormone, it also inhibits its peripheral conversion. 
lodine therapy to inhibit the synthesis of thyroid hormones should be performed one hour after the administration of PTU, thus preventing the use of iodine as substrate.

In cases of thyrotoxic storm, higher doses of PTU are necessary. Propranolol is the beta-blocker of choice, since in addition to its cardiovascular effects, it also inhibits peripheral conversion of thyroid hormones. Other beta-blockers are not contraindicated. The level of cortisol tends to be normal, but the use of corticosteroids is indicated to achieve levels compatible with stress. Publications have reported the use of plasmapheresis in the preoperative preparation in selected cases ${ }^{11}$. Elective procedures should be postponed until thyroid hormones are under control.

The early onset of prenatal care and routine use of ultrasound contribute to the early diagnosis of GTD. Thus, clinical presentations with large hydatidiform moles, elimination of vesicles, anemia, and emergency situations have become increasingly less common. 